\title{
http:// bjas.bu.edu.eg \\ Pining Fixation Versus Canulated Screw with Open Reduction of Pediatric Lateral Condyle Humerus Fractures
}

\author{
M.E.A.Aamer ${ }^{1}$, H.A.Bassiooni ${ }^{2}$, A.S.El Gazar ${ }^{2}$ and A.S.El-Hammady ${ }^{2}$ \\ ${ }^{1}$ Orthopedic Surgery Dept., El- Sahel teaching hospital, Cairo, Egypt \\ ${ }^{2}$ Orthopedic surgery Dept., Faculty of Medicine, Benha Univ., Benha, Egypt \\ E-Mail: mido_aamer@yahoo.com
}

\begin{abstract}
Good results have been described for lateral condyle Fractures pediatrics treated by open reduction and fixation using Kirschner wires or screws. We, in our level III retrospective comparison clinical research study, retrospectively reviewed 20 patients (10 K-wire, 10 screw fixation; average age 5.6 years, average follow-up 6.8 months). The clinical outcomes were evaluated according to the criteria of Hardacre et al. There was no statistically significant difference in clinical outcome between these two groups. Two patients developed skin infection around K-wires, while no infection occurred in fracture with screws. An obvious lateral prominence occurred in one patient with K-wires and none with screws. One patient with K-wires and one patient with screws had a lack of $15^{\circ}$ of extension of the elbow compared with the other side. With K-wires there were two nonunions (average time to union 9.6 weeks). With screws, one nonunions (average of 6.8 weeks). Both K-wires and cannulated screw fixation are effective in treatment for lateral humeral condyle fracture. K-wires can pass through the ossific nucleus of capitulum without damaging it, but a longer period of external fixation and local skin care will be required. Screw fixation patients spent fewer days in a cast and had agreater range of motion at the last follow-up. Screw fixation is associated with fewer nonunions and faster time to union, but a secondary procedure for removal is required .
\end{abstract}

Keywords: Fixation, lateral condyle, Open reduction,canulated screw , K.wire.

\section{Introduction}

Pediatric lateral condyle distal humeral fractures, the 2nd most common elbow fracture that involves the growth plate, account for $10 \%$ to $15 \%$ of all pediatric fractures of the elbow, with a high incidence between 4 and 10 years of age[1,2].These injuries are typically the result of an avulsion of a portion of the humeral condyle by pull of the extensor musculature due to a Varus force on a supinated forearm or by the direct force of the radial head onto the condyle in the setting of a fall and axial load through an extended elbow.[3]The child with a lateral condyle fracture will usually present with lateral elbow pain and a limited range of elbow motion[4].Unlike supracondylar fractures, lateral condyle fractures may not be associated with an obvious swelling or deformity. A high index of clinical suspicion and appropriate radiographic investigations are required to recognize the more subtle fracture patterns[5].Undisplaced fractures may not be easily detectable on standard anteroposterior and lateral radiographs therefore the addition of oblique views has been advocated[6]. Multiple treatment options are available for these fractures, ranging from simple immobilization for nondisplaced or minimally displaced fracture patterns, to operative reduction and fixation with Kirschner wires (K-wires) or screws for displaced fracture [7].Although some controversy exists with regard to the acceptable amount of displacement, fractures with displacement greater than 2 or $3 \mathrm{~mm}$ are generally thought to require reduction and fixation to facilitate union and prevent deformity and articular incongruity [8].Because of concerns about the possibility of loss of fixation with brief use of K-wires or occurrence of infection with their prolonged use,WP. Loke, MH.Shukur and JK. Yeap have explored the use of screw fixation for lateral condyle fractures[8].The fractures have a high risk for complications including nonunion, malunion, ulnar nerve paresis, hypertrophic scar, avascular necrosis of ossific nucleus and angular deformity[9].

\section{Materials and methods}

Aretrospective review of 20 patients with displaced lateral condyle fractures with varying degrees of displacement was performed from 2016 to 2019 in ElSahel Teaching Hospital patients with age from 2 year to 8 year. Patients were treated by open reduction and $\mathrm{K}$.wires or $4 \mathrm{~mm}$ cannulated screw fixation.The clinical records and radiographs of patients were retrospectively analysed. Fractures were classified according to the Milch system [10]. All patients underwent anteroposterior and lateral radiographs, and the displacement of the fracture fragment in radiographs was measured and recorded.The fracture gap was measured from lateral metaphyseal cortex of the distal humerus to the lateral cortex of the fracture fragment.The greatest displacement on any single radiograph was considered as the amount of displacement of the fracture.The demographic data and follow-up information are summarized in statistically analysis. Informed consent from all patients and patients' parents or guardians was obtained. Atraditional lateral approach to the elbow was performed following general anaesthesia.Care was taken to limit damage to the soft tissue and periosteum. After open reduction of the fracture, K-wires or cannulated screws were used to stabilize the fracture. K.wires or screws were required to pass through the metaphyseal fragment.If there was not enough space in the metaphyseal fragment, K-wires or screws might pass through the ossific nucleus of the capitulum.All K-wires were placed percutaneously into the fractures. After operation, an above elbow slab was used to support the fracture fixed with K-wires for five or six weeks. When the fracture united, the percutaneous $\mathrm{K}$ wires were removed at an outpatient clinic without anaesthesia six or eight weeks after the operation.The 
fracture with screws was supported by an above elbow slap for one or two weeks. After the slap was removed, the patient was required to do exercises of the elbow. When radiographs showed sufficient evidence of fracture union, screws were routinely removed at eight or 12 weeks in the operating room under general anaesthesia. An independent observer reviewed all records of charts and radiographs in order to ensure the uniformity of analysis. The clinical information in the two groups was recorded including the patient's age at injury, the type of fixation, affected side, the period of K-wires or screw fixation, the functional result of the elbow and complications.In addition, radiographic information including the union of fractures, nonunion, malunion or lateral prominence was recorded for the radiographic evaluation. The clinical results were evaluated according to the criteria suggested by Hardacre et al[11].This study was performed in accordance with the ethical Standards of ethical committee.

\section{Statistical analysis}

The demographic (age and sex) and clinical characteristics of the treatment group (follow-up in months, union, time to union, days in cast) were compared using Student's t-tests and $\chi 2$-tests for continuous and categorical variables, respectively. For categorical variables with expected counts less than 5, Fisher's exact test was used. Median values for range of motion for flexion, extension, and total arc were compared using Wilcoxon's rank-sum test; in addition, the proportion of patients with loss of extension greater than 10 and flexion less than 120 were compared using Fisher's exact tests.

\section{Results}

Twenty patients were identified and formed the study group. Demographic and clinical characteristics have been summarized.Fractured classified by Milch classifications, all patient operated with screw were type II but in patients operated by k. wires were $80 \%$ Milch I and $20 \%$ type II . Two K-wires were used in cases ,but in case of screw fixation using single cannulated $4.0 \mathrm{~mm}$ or $4.5 \mathrm{~mm}$ were used. The screw was typically placed through the metaphyseal portion of the lateral condyle, rather than through the ossific nucleus of the capitellum. Patients with fractures repaired with screw fixation spent fewer days in a cast and had a shorter time to union ( 7.45 vs. 8.5 weeks). Three delayed unions (healing at 3-6 months) and one nonunion (not healed at greater than 6 months) (reoperated using bone graft and pin fixation) in the K-wire fixation group were observed. In the screw fixation group, one delayed union was observed (healed at 12 weeks). No nonunion were observed in the screw fixation group. Patients with screw fixation had a statistically significantly greater median flexion and total arc of motion. The number of patients who had a clinically significant range of motion deficit with follow-up of at least 3 months did not differ [ $>100$ loss of extension one in screw fixation and three in pin fixation, $<120$ flexion (one in screw fixation and three in pin fixation). According to classification of outcomes using the Hardacre criteria. In the screw fixation group 7 results $(70 \%)$ were excellent, two (20\%) were good and one were poor due to revision. In the K-wire group, 6 results were excellent $(60 \%), 3 \operatorname{good}(30 \%)$ and one poor $(10 \%)$ due to non-union. Complications were observed in 7 patients in the K-wire fixation group (70\%) and three patients in the screw fixation group (30\%). In addition to the delayed union and nonunion cases in the $\mathrm{K}$ wire group, complications included two case of pin-tract infection, one fishtail deformity. One additional secondary procedures were performed in the K-wire group for grafting of nonunion. In the screw fixation group, the delayed union not required surgical revision. Other complications included one case of loss of reduction at the 3rd day of operation and required revision using pin fixation, and one case in which the screw backed out without loss of reduction, which was treated by screw removal at 6 week.

\section{Discussion}

Fractures of the lateral condyle of the humerus represent the second most common type of fracture of the elbow in children.Although this injury is seen at all ages, it is predominately observed in children aged between 5 and 10 years. These injuries are typically the result of an avulsion of a portion of the humeral condyle by pull of the extensor musculature due to avarus force on a supinated forearm or by the direct force of the radial head onto the condyle in the setting of a fall and axial load through an extended elbow. Multiple treatment options are available for these fractures, ranging from simple immobilization for nondisplaced or minimally displaced fracture patterns, to operative reduction and fixation with Kirschner wires (K-wires) or screws for displaced fractures.

Although some controversy exists with regard to the acceptable amount of displacement, fractures with displacement greater than 2 or $3 \mathrm{~mm}$ are generally thought to require reduction and fixation to facilitate union and prevent deformity and articular incongruity.

Controversy exists with regard to the optimum management of lateral condyle fractures in children.We sought to examine whether screw fixation of displaced lateral condyle fractures would decrease time to union.

Limitations include the retrospective design, resulting in inconsistent reporting of range of motion, and the short follow-up period. The short duration of follow-up limits interpretation of the range of motion data and Hardacre outcome. In addition, the screw fixation group tended to be treated more recently, possibly accounting for some of the variability in casting duration.

Although using K-wires may be a relatively simpler method of fixation, outcomes may be limited by the propensity for wire loosening/ migration and the duration of immobilization needed to achieve adequate healing.

An additional consideration with pin fixation is whether to leave the pins exposed (with the possibility of developing pin-site infection that can progress to deep infection)or to bury the pins beneath the skin (resulting in the need for a secondary procedure for pin removal). 
As an alternative, screw fixation affords the opportunity to apply compression across the fracture and to initiate range of motion while the fixation is still in place.The median values for range of motion were increased in the screw fixation group,but the rate of clinically significant loss of range of motion was not different between the two groups.

Concerns exist with regard to growth disturbance,but some series have suggested that screw fixation may actually decrease lateral overgrowth [12-13].

The current follow-up is insufficient to determine whether growth disturbance may be problematic. One other important consideration when screw fixation is utilized is the additional cost of a secondary procedure for screw removal.

In our K-wire fixation group, one patients underwent procedures for bone graft for non-union.

In contrast, screw removal has been recommended for all patients in the screw fixation group. The added costs for implants and secondary procedures for screw removal would likely be only partially offset by costs associated with infection, non- union and pin removal in the Kwire group and would likely result in additional costs to the health care system with a screw fixation strategy.

In a study by $\mathrm{Li} \mathrm{WC}, \mathrm{Xu} \mathrm{RJ}$. reported the results of screw fixation (32 patients) compared with $\mathrm{K}$-wire fixation (30 patients). They observed no significant difference in clinical outcome and no nonunion.They reported more frequent limitation of motion and a higher rate of infection in the $\mathrm{K}$-wire group.They observed clinically apparent lateral overgrowth in $37 \%$ of patients treated with K-wires and $12 \%$ of those treated with screws. They also observed differences in the carrying angle in $23 \%$ of patients treated with K-wires(six of seven of these appearing to have cubitus varus), whereas $19 \%$ of screw fixation patients had apparent cubitus valgus[14].

Results of K-wire fixation were reported by V.Boz, AE.Ulusal , H.Vuruskaner, Y.Aydinoglu who observed excellent functional results at an average follow-up of 39 months in $71.3 \%$ of 69 patients. Of the patients, $47 \%$ were observed to have lateral condylar overgrowth.Nonunion, however, was not observed in any patient [15].

In a study by DP.Thomas, AW.Howard, WG.Cole, DM.Hedden 104 patients underwent a 3-week period of $\mathrm{K}$-wire fixation,with one instance of nonunion,two cases of infection, and a $44 \%$ incidence of abnormal elbow shape on late review(which included lateral condylar overgrowth and excessive bone formation over the outer surface of the condyle[16].

In a study by SV.Skak, SD.Olsen, Smaabrekke describe the use of K-wire and Palmer nail fixation in 21 patients, reporting that radiographically healed at all but one case was a later review.It was documented that, in all of these patients, the distal humerus was wider in comparison with the unaffected side following healing. Two of these patients subsequently developed avascular necrosis of the trochlea [17].

Of 16 fractures treated with $\mathrm{K}$-wire fixation by M.Jenyo , T.Mirdad. malunion was observed in one case,resulting from loosening of a K-wire, and non union was observed in one case, requiring subsequent bone grafting and screw fixation[18].

In a study by Weiss observed no non union and a $3.8 \%$ infection rate in the entire group of patients treated with K-wire fixation and 4 weeks of cast fixation. Among the 73 patients who had displacement requiring open reduction, nine had malunion, loss of reduction or nonunion[19].

In another large series of 105 patients with K-wire fixation following open reduction for displaced fractures done by A.Leonidou, K.Chettiar, S.Graham, P.Akhbari, K.Antonis, E.Tsiridis, O.Leonidou. reported $96 \%$ excellent results with no non-unions, no loss of motion and four patients with cubitus varus [20].

With regard to screw fixation,WP.Loke, MH.Shukur, JK.Yeap examined 34 patients with an average follow-up of 24.5 months and found the average time to radiographic union to be 6.9 weeks. Excellent functional results were observed in $82 \%$. Lateral overgrowth was observed in two patients, lateral condylar avascular necrosis resulting in a valgus deformity in two patients, and a fishtail deformity in three patients[5].

In a study by JC.Sharma, A.Arora, NC.Mathur, SP.Gupta, A.Biyani, R.Mathur followed up 37 children in whom $4.0 \mathrm{~mm}$ cancellous lag screws were placed with a mean follow-up of 4.8 years.Painless, full range of motion was observed in 36 of the 37 patients. The one outlying patient was observed to have a delayed union with loss of $10^{\circ}$ of elbow motion in comparison with the contralateral side. Mild fishtail deformity was also observed in three of the cases,but this was not observed to be functionally relevant.There was no instance of nonunion, avascular necrosis, or premature epiphyseal fusion [4].

In a study by Hasler and von studied 66 fractures, of which 27 were treated with metaphyseal lag screw placement.At a mean follow-up of 10 years, all 27 of the operative cases demonstrated anatomic union, symmetric carrying angles with the unaffected side, and full range of motion [11].

\section{Conclusion}

Surgeons currently treat lateral condyle fractures with K-wires or screws, but only one smaller prior study compared the two approaches. Our study supports that screw fixation is a valuable, with no non-unions, faster time to union and fewer complications than pin fixation. These potential advantages must be weighed against the need for subsequent screw removal.Longer follow-up will be required to assess effects on growth.

\section{References}

[1] E.Shirley, M.Anderson, K.Neal Screw fixation of lateral condyle fractures: results of treatment .J., Pediatr Orthop,Vol. 35,PP.8214,2015.

[2] C.Flynn, JF.Richards, RI.Saltzman; Prevention and treatment of non-union of slightly displaced fractures of the lateral humeral condyle in children. An endresult study. J Bone Joint Surg Am ,Vol.57,PP.10871092,1975 . 
[3] RT.Morrise, SL.Weinstein; Lovelland Winter's pediatric orthopaedics, $6^{\text {th }}$ edition. Philadelphia,PA :Lippincott ,Williams , and Wilkins;vol 2, PP.14541457,2006.

[4] JH. Beaty and JR. Kasser:The elbow region: general concepts in the paediatric patient. In: JH. Beaty andJR. Kasser ,Rockwood and Wilkins' fractures in children. Philadelphia, PA: Lippincott Williams \& Wilkins, PP.475-486,2010.

[5] MS.Gaston, GR.Irwin and JS. Huntley ; Lateral condyle fracture of a child's humerus: the radiographic features may be subtle. Scot Med J., Vol. 57,PP. 182,2012.

[6] KS.Song, CH.Kang, BW. Min, KC. Bae and CH.Cho; Internal oblique radiographs for diagnosis of nondisplaced or minimally displaced lateral condylar fractures of the humerus in children. J Bone Joint Surg Am,Vol. 89,PP. 58-63,2007.

[7] JC.Sharma, A.Arora, NC.Mathur, SP.Gupta, A.Biyani , R.Mathur ;Lateral condyle fractures of the humerus in children:fixation with partially threaded $4.0 \mathrm{~mm} \mathrm{AO}$ cancellous screws .JTrauma , Vol.39,PP.1129-1133, 1995.

[8] WP.Loke, MH.Shukur, JK.Yeap.;Screw osteosynthesis of displaced lateral humeral condyle fractures in children:amid-term review.Med J Malaysia ,Vol.61,PP.40-44,2006.

[9] S. Toh, K.Tsubo, S.Nishikawa, S.Inoue, R.Nakamura, S.Harata : Long-standing nonunion of fractures of the lateral humeral condyle. J Bone Joint Surg Am,Vol. 84-A(4),PP.593-598,2002.

[10] H.Milch : Fractures and fracture dislocations of the humeral condyles. J Trauma,Vol.4,PP.592-607,1964.

[11] A.Hardacre, SH.Nahigian, AI.Froimson, JE.Brown : Fractures of the lateral condyle of the humerus in children. J Bone Joint Surg Am ,Vol.53(6),PP.10831095,1971.

[12] Awaya, Hitomi, Schweitzer, E.Mark, Feng, A.Sunah, Kamishima, Tamotsu, Marone, J.Phillip, Farooki, Shella, Trudell, J.Debra, Haghighi, Parviz, Resnick, L.Donald : Elbow Synovial Fold Syndrome: MR Imaging Findings". American Journal of Roentgenology,Vol.177(6),PP.1377-1381, 2001.

[13] WE. Yang, CH.Shih, ZL.Lee: Anatomic reduction of old displaced lateral condylar fractures of the humerus in children via a posterior approach with olecranon osteotomy. J Trauma,Vol.64,PP.1281-1289,2008.

[14] Li WC, Xu RJ :Comparison of Kirschner wires and AO cannulated screw internal fixation for displaced lateral humeral condyle fracture in children. Int Orthop ,Vol.36 , PP. 1261-1266,2012.

[15] V.Boz, AE.Ulusal, H.Vuruskaner, Y.Aydinoglu: Functional results of displaced lateral condyle fractures of the humerus with 4 week K-wire fixation in children. Acta Orthop Trauamatol Turc, Vol. 39,PP.193-198,2005

[16] DP.Thomas , AW.Howard, WG.Cole, DM.Hedden : Three weeks of K-wire fixation for displaced lateral condyle fractures of the humerus in children. J Pediatr Orthop, Vol. 21,PP.565-569,2001.

[17] SV.Skak, SD.Olsen, A.Smaabrekke :Deformity after fracture of the lateral humeral condyle in children. $J$ Pediatr Ortho, Vol. 10,PP.142-152,2001.

[18] M. Jenyo and T. Mirdad :Fractures of the lateral condyle of the humerus in children. East Afr Med J,Vol. 78,PP.424-429,2001.

[19] JM.Weiss, S.Graves, S.Yang, E.Mendlesohn, RM. Kay, DL.Skaggs: A new classification system predictive of complications in surgically treated pediatric humeral lateral condyle fractures. J Pediatr Orthop.,Vol. 29,PP.602-605,2009.

[20] A.Leonidou, K.Chettiar, S.Graham, P.Akhbari, K.Antonis, E.Tsiridis, O.Leonidou :Open reduction internal fixation of lateral humeral condyle fractures in children. A series of 105 fractures from a single institution. Strategies Trauma Limb Reconstr., Vol.9, PP.73-78,2014. 